\title{
Cooperation of the haves and the have-nots
}

\author{
Kaumudi H Prabhakara, ${ }^{1,2}$ Albert J Bae, ${ }^{2}$ and Eberhard Bodenschatz ${ }^{1,2}$ \\ ${ }^{1}$ Laboratory of Atomic and Solid State Physics, \\ Department of Physics, Cornell University, Ithaca, USA \\ ${ }^{2}$ Max-Planck Institute for Dynamics and Self-Organization, Göttingen, Germany
}

(Dated: December 9, 2017)

\begin{abstract}
Upon starvation, Dictyostelium discoideum (D.d.) exhibit social behavior mediated by the chemical messenger cyclic adenosine monophosphate (cAMP). Large scale cAMP waves synchronize the population of starving cells and enable them to aggregate and form a multi-cellular organism. Here, we explore the effect of cell-to-cell variability in the production of cAMP on aggregation. We create a mixture of extreme cell-to-cell variability by mixing a few cells that produce cAMP (haves) with a majority of mutants that cannot produce cAMP (have-nots). Surprisingly, such mixtures aggregate, although each population on its own cannot aggregate. We show that (1) a lack of divalent ions kills the haves at low densities and (2) the have-nots supply the cAMP degrading enzyme, phosphodiesterase, which, in the presence of divalent ions, enables the mixture to aggregate. Our results suggest that a range of degradation rates induces optimal aggregation. The haves and the have-nots cooperate by sharing complementary resources.
\end{abstract}




\section{INTRODUCTION}

Dictyostelium discoideum (D.d.) is a soil-dwelling amoeba, about $10 \mu \mathrm{m}$ in size with a unique survival strategy. In nutrient rich environments, the cells feed, divide and lead a solitary life, but when food becomes scarce and the cells start to starve, they exhibit social behavior $[1,2]$. D.d. signal each other by generating excitable waves of the chemical messenger cyclic adenosine monophosphate (cAMP)—when cells at one location sense that the external cAMP concentration is rising due to secretions from their neighbors to one side, these cells are in turn stimulated to secrete cAMP and relay the signal to their neighbors to the other side. To bring the concentrations back down after a wave passes, there is global degradation of cAMP by a phosphodiesterase (PDE) that is continuously secreted by the cells $[3,4]$. Excitation centers emerge, and after about 6 hours of starvation, the cells become chemotactic, and migrate towards these centers. Approximately $10^{5}$ cells come together to form a multi-cellular organism which culminates into a fruiting body composed of a ball of spores atop a stalk. When food is restored, the spores germinate into amoebae. This unique ability of $D . d$. to transform from solitary cells to a multi-cellular organism has made it a much studied model system for morphogenesis [5-8].

Essential to the survival of D.d. is its ability to self-organize by chemical signaling. However, in a population, there is significant cell-to-cell variability in cAMP production [911]. How then is the signaling mechanism so robust? We intensified the cell-to-cell variability by mixing two kinds of cells, those that can produce cAMP, which we call the haves, and the have-nots, which are mutants that cannot produce cAMP and thus cannot aggregate. Puzzlingly, when we add a small number of haves to a high density of have-nots, the mixture aggregates, whereas neither population aggregates on its own. In this paper we solve this puzzle.

\section{RESULTS}

Development of D.d. under starvation is typically studied at densities of $\approx 10^{5}$ cells $/ \mathrm{cm}^{2}$ on agar $[6,12]$. In our experiments, we worked with similar densities of cells, albeit on a Petri dish under a layer of buffer solution instead of agar, because this gave us the greatest uniformity in cell plating density. At these densities, cells initially form a confluent layer 


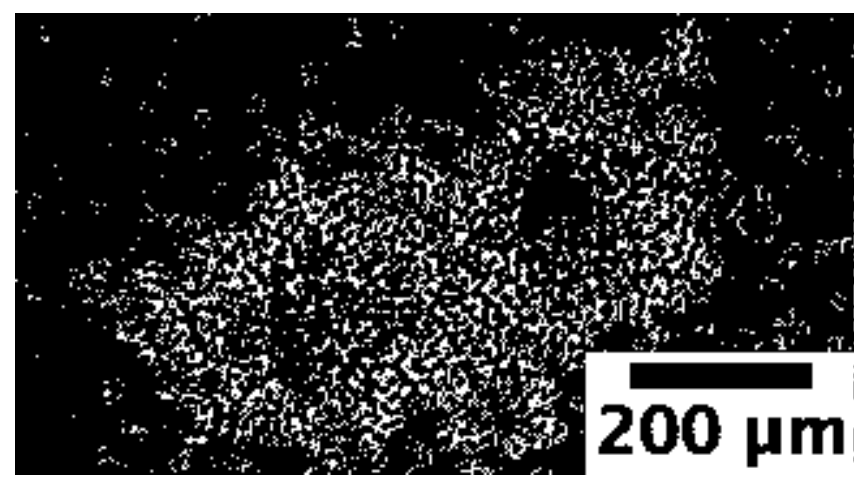
aggregation. movie S1).

on the surface and later aggregate under starvation. When we decreased the density below $\approx 10^{4}$ cells $/ \mathrm{cm}^{2}$, the population no longer aggregated. However, when we added about $10^{5}$ cells $/ \mathrm{cm}^{2}$ of the acaA- mutants (have-nots) to these low densities of wild-type cells (haves) aggregation was rescued (figure 1). This is surprising because a pure population of $10^{5}$ cells $/ \mathrm{cm}^{2}$ of have-nots didn't aggregate, as they were unable to produce cAMP. Since neither the low-density population of haves nor the high-density of have-nots was capable of aggregating on their own, there must be a synergy between the two populations that rescues

How do these haves and have-nots cooperate? The cooperation between the two populations can only be chemical or mechanical in nature. Let us first hypothesize that the presence of have-nots is sensed by haves through mechanical interactions because the havenots don't produce cAMP, and therefore shouldn't directly affect the cAMP relay. This mechanical hypothesis is supported by recent work showing that mechanical stresses play a role in signal transduction $[13,14]$. To first approximation, we replaced the mutants with $10 \mu \mathrm{m}$ polystyrene beads. If the interactions were due to simple mechanical contact, beads might play the role of the have-nots. This, however, we did not observe (in two experiments). The beads did not rescue aggregation. As a positive control, to ensure the beads are not harmful to the cells, we added the beads to a high density of haves, where we know the cells do aggregate. We found that the cells not only aggregated, but also actively transported the beads as they moved and incorporated them into their aggregates (figure 2, Supplementary

FIG. 1. (a) A mixture of the high-density have-nots and a low-density haves can aggregate, whereas (b) a low-density population of haves cannot aggregate on its own. Images were taken about $12 \mathrm{~h}$ after start of starvation. These experiments were repeated seven times. 


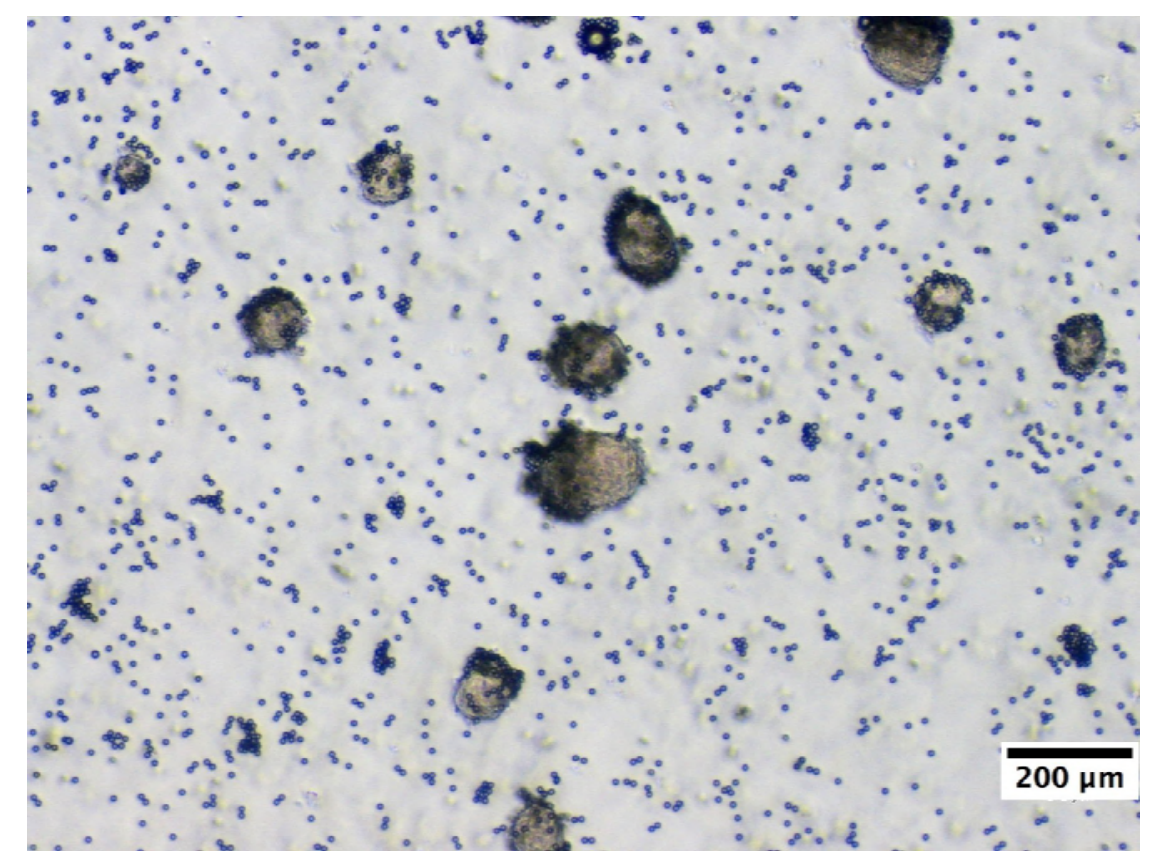

FIG. 2. Beads added to a high-density population of haves are incorporated into the aggregates. These experiments were performed about 35 times with different ratios of the beads to the highdensity haves. The beads did not affect pattern formation in all cases.

Other subtler contact-dependent interactions could be in play. To completely rule these out, we placed a Millipore filter between the populations of the haves and the have-nots. This filter allows the exchange of chemicals, while preventing the cells from coming into mechanical contact. The populations aggregated (in two trials of the experiments). Thus, we have disproved our mechanical hypothesis. The effect must be chemical in origin.

What is this chemical interaction? Could the aggregation be caused by some yet-to-beidentified chemical factors secreted by the high-density cell populations into the buffer? We obtained supernatants from three sources: a high-density population $\left(2 \times 10^{5}\right.$ cells $\left./ \mathrm{cm}^{2}\right)$ of haves, a high-density population of have-nots $\left(2 \times 10^{5}\right.$ cells $\left./ \mathrm{cm}^{2}\right)$, and the mixture described above $\left(9 \times 10^{3}\right.$ cells $/ \mathrm{cm}^{2}$ of wild type cells and $2 \times 10^{5}$ cells $/ \mathrm{cm}^{2}$ of acaA- mutants). We starved these populations for six hours and then carefully collected the supernatants. Each of these supernatants was added to a low-density population of haves. Aggregation was rescued in all three cases (in three repetitions of the experiment), showing that the factors responsible for aggregation are secreted by both haves and have-nots.

What in the supernatants causes the aggregation? To narrow down the list of chemical factors that could potentially be responsible, we separated the supernatant into two fractions, 


\section{Supernatant}

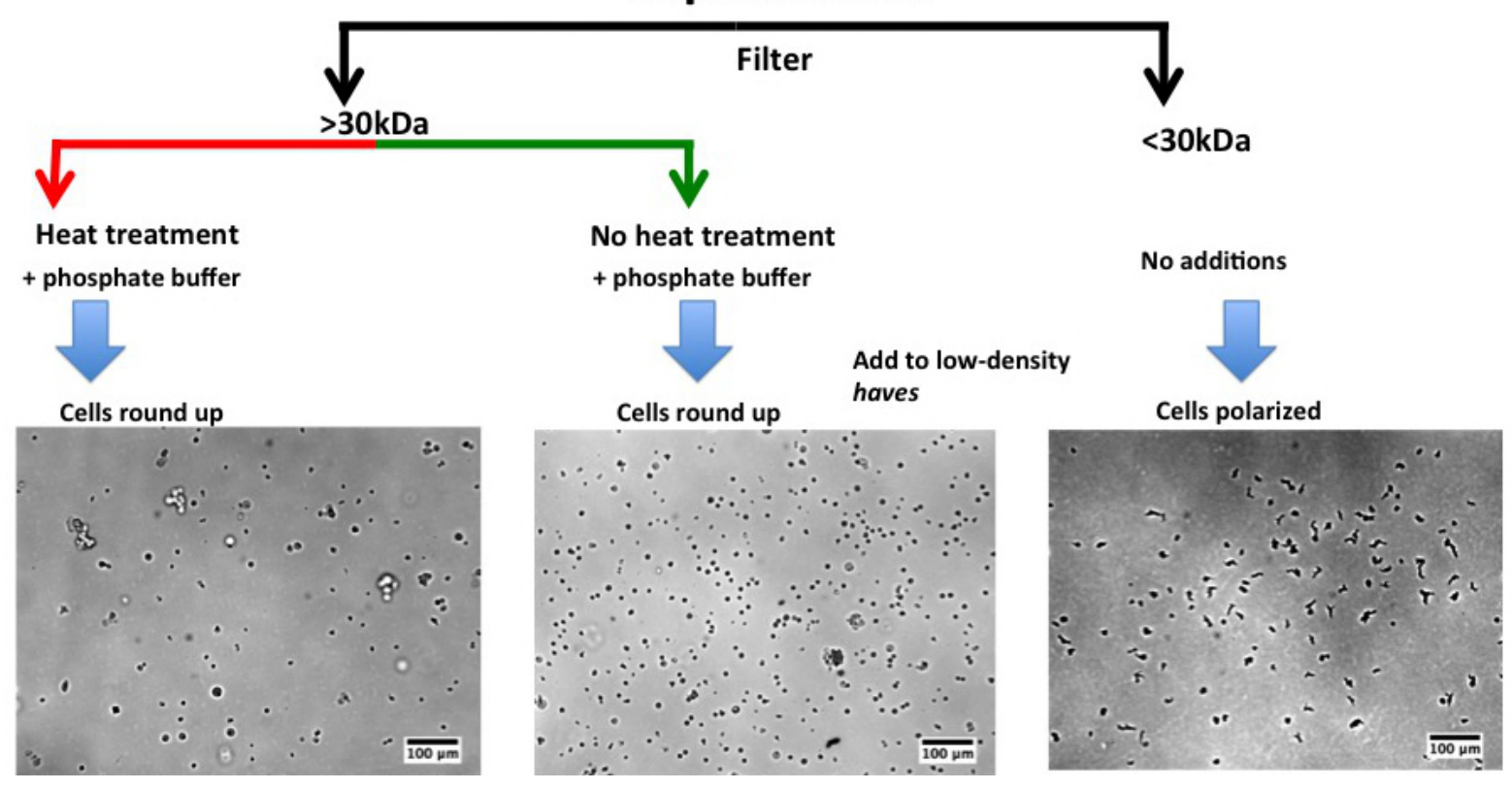

FIG. 3. Supplementation of the low-density haves with different components of the supernatant.

Adding just the HMWF of the supernatant, both heat-treated and non-heat treated, caused the cells to round up. When the LMWF of the supernatant was added, the cells were healthy and polarized.

one containing chemicals $>30 k D a$ and a fraction $<30 k D a$, containing small proteins, ions, and other low molecular weight chemicals (see Methods). We denote these fractions as the High Molecular Weight Fraction (HMWF) and Low Molecular Weight Fraction (LMWF) respectively. To further fractionate the HMWF fraction, we subjected a part of it to heat treatment to deactivate heat sensitive enzymes, and left the rest untreated.

Low densities of haves were developed in these fractions. After about 20h, we found (in one experiment):

- Cells + LMWF: The low-density haves were viable and appeared polarized, but no tight aggregates were observed. (figure 3)

- Cells + heat-treated, HMWF: The low-density haves rounded up and did not appear viable. Furthermore, many cells had de-adhered from the substrate (figure 3).

- Cells + non-heat-treated, HMWF: The low-density haves also rounded up and did not appear viable (figure 3 ). 


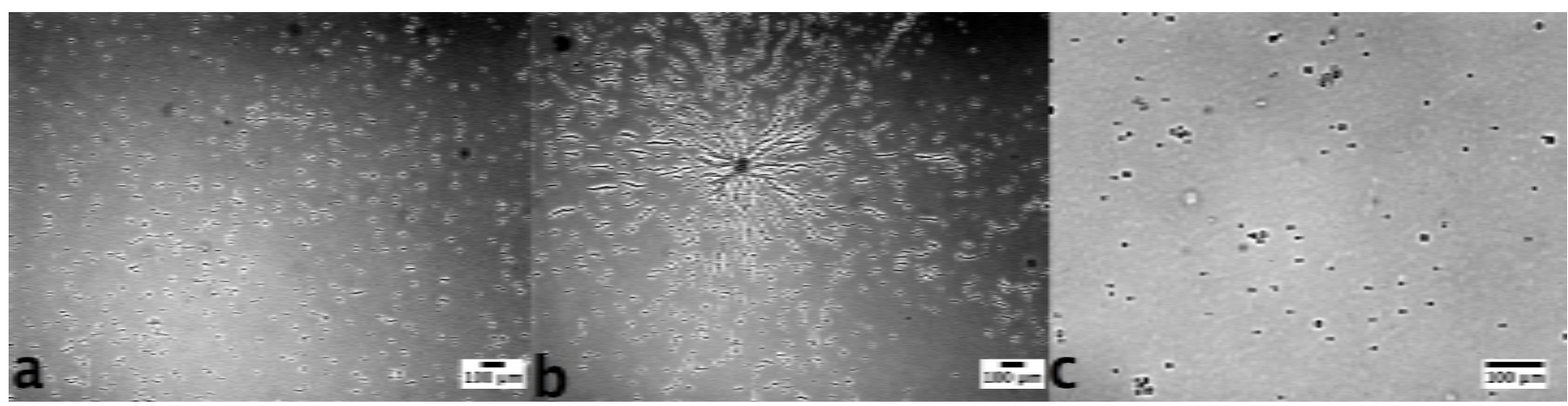

FIG. 4. (a) Positive control - heat-treated, HMWF of the supernatant mixed with the LMWF was used to condition the low-density haves. (b) Positive control - non-heat treated, HMWF of the supernatant mixed with the LMWF was used to condition the low-density haves. (c) Negative control - buffer was added to the low-density haves.

- Cells + LMWF + heat-treated, HMWF: In this positive control, the low-density haves looked polarized (figure 4a).

- Cells + LMWF + non-heat-treated, HMWF: In this second positive control, the lowdensity haves showed clear large-scale streaming (figure 4b).

We also conducted a negative control, in which the low-density haves were unconditioned. These cells rounded up and did not appear viable (figure 4c). Clearly, something smaller than $30 \mathrm{kDa}$ is essential to maintain viability. This chemical is secreted by both haves and have-nots, and is deficient in our buffer. Additionally, a heat-sensitive factor larger than $30 \mathrm{kDa}$ enables the cells to signal effectively over long distances.

What could be in the LMWF that keeps the cells alive? We hypothesize that cells carry over essential ions into our low ionic strength buffer. At low densities the ionic strength might not be sufficient for the cells to remain viable [15-17]. To test this hypothesis, we supplemented our buffer with either $50 \mu \mathrm{M} \mathrm{CaCl}_{2}, 50 \mu \mathrm{M} \mathrm{MgCl}_{2}$ or $150 \mu \mathrm{M} \mathrm{NaCl}$. For the latter, we used a higher concentration to match the ionic strengths of the other two buffers. In the calcium and magnesium enriched buffers, (figures 5a,b) after $20 \mathrm{~h}$, the low-density haves were viable and polarized, but did not form clear aggregates. In contrast, the lowdensity haves in the sodium enriched buffer (figure 5c) rounded up, indicating that they were not viable. We can conclude that divalent ions, $\mathrm{Ca}^{2+}$ or $\mathrm{Mg}^{2+}$, which are secreted by have-nots as well, are required to keep the cells viable. 


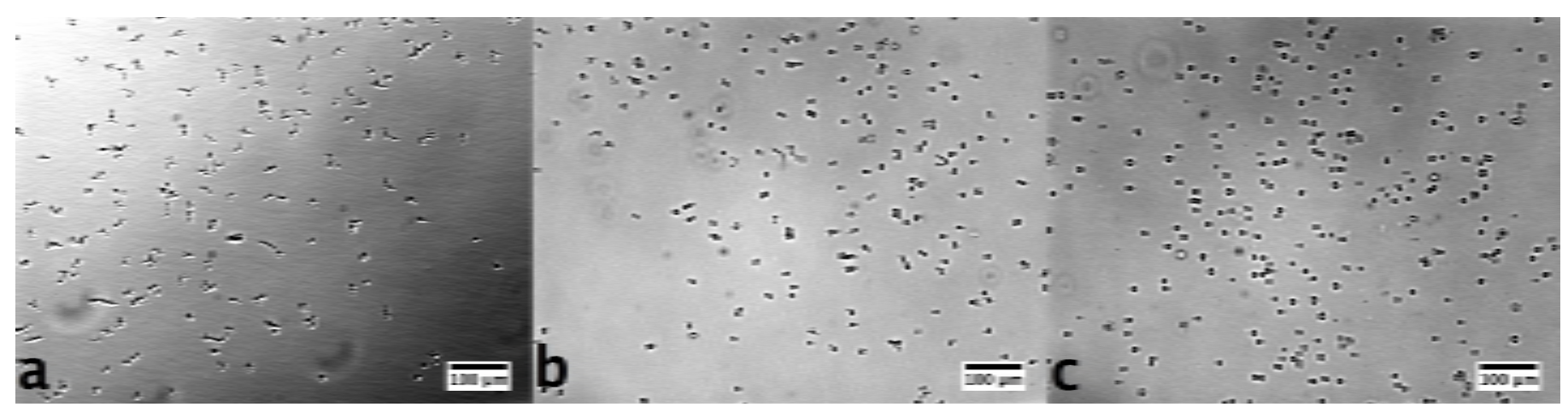

FIG. 5. Low density haves in (a) calcium enriched buffer were polarized and beginning to stream, (b) magnesium enriched buffer were polarized and beginning to stream, (c) sodium enriched buffer rounded up and were therefore unhealthy. Images were taken a day after start of starvation. The experiments with calcium were performed five times, while the experiments with sodium and magnesium were performed once.

Although the addition of calcium and magnesium kept the cells viable, it did not fully rescue aggregation. This is consistent with the experiment where we added the LMWF of the supernatant to the low-density haves. Above, we showed that factors in the heat-sensitive HMWF were necessary to rescue aggregation (figure 4a,b). In the HMWF, we expect to find Conditioned Medium Factor (CMF) and PDE, both of which were shown to be important for aggregation [18-21]. PDE is heat-sensitive [22-24] (see supplementary note S1 for the experimental verification), whereas CMF is not [18]. Could PDE be the missing factor? To demonstrate that this is indeed the case, we added exogenous PDE (PDE4A) at various concentrations to the low-density haves conditioned by calcium enriched buffer. We found that at concentrations of PDE above 2 units/ml (see Methods), normal aggregation behavior was recovered (figure 6).

\section{DISCUSSION}

We opened this manuscript with a riddle: when a low-density population of haves were added to a high-density population of have-nots, the mixture aggregated, whereas each population on its own fails to develop.

We found that the lack of divalent ions kills the low-density haves. Ions like $\mathrm{Ca}^{2+}$ and $\mathrm{Mg}^{2+}$ are necessary to keep the cells viable, but they alone do not enable the low-density 


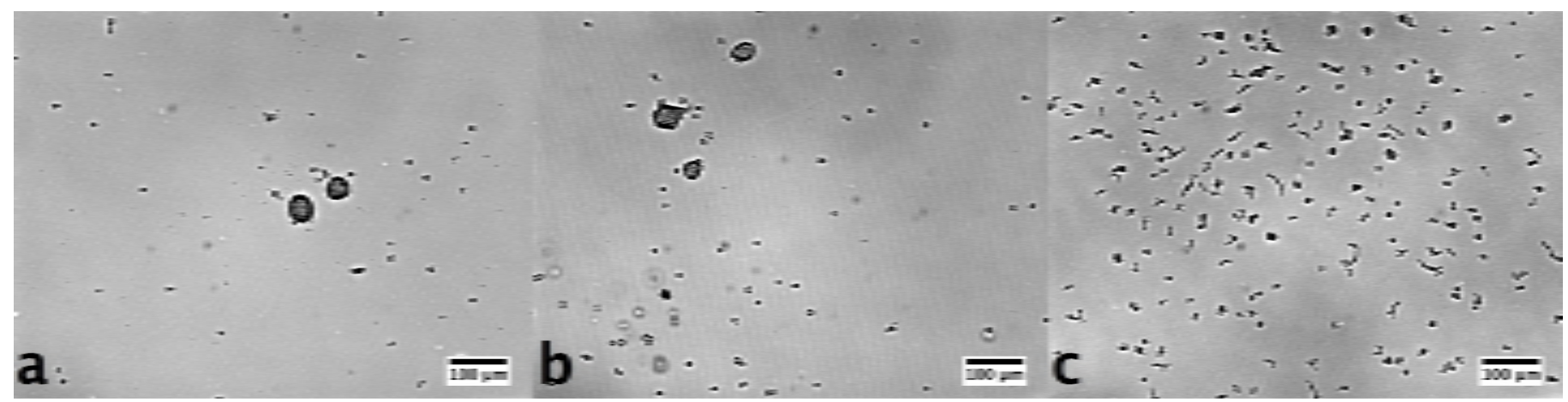

FIG. 6. Addition of PDE in different amounts to calcium enriched buffers to condition the low density WT cell populations. (a) 49 units/ml (b) 2 units/ml (c) 0.1 units/ml. Images were taken a day after start of starvation. Experiments were performed once.

haves to form tight aggregates. Addition of external PDE is necessary for these low-density haves to aggregate. Although addition of calcium does induce the cells to secrete more PDE (see Supplementary note S1 and [25-28]), the increased PDE activity due to the addition of calcium is not sufficient to fully restore aggregation in a population of low-density haves.

We found that the addition of external PDE to the low-density haves in buffers enriched with divalent ions, enabled them to form tight aggregates. Previously, it was shown that

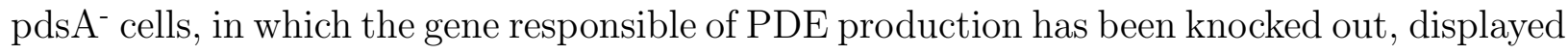
decreased PDE activity and aberrant development and chemotaxis [29-31].

The importance of PDE can be understood by considering wave propagation in populations of D.d. The propagation of more than one wave-front requires degradation of cAMP within one wave period, i.e., within about 6 - 8 min, most of the cAMP should be degraded. As an estimate, if the cells communicate over a length scale corresponding to about 10 cell lengths, which is about $130 \mu \mathrm{m}$ at high cell densities, the required degradation rate is about 1.4/ min, which corresponds to 0.7 min. (See Appendix A1 for more details.) Being much smaller than the wave period, this degradation time allows wave propagation, which we observe in the mixture of haves and have-nots (see supplementary movie S2). If we assume that degradation rate scales linearly with density, then at the low cell density we consider, the degradation rate is about $50 \mathrm{~min}$. This is much longer than the wave period. Therefore well-developed spirals cannot exist, which agrees with what we observe in the low-density population of haves in a calcium enriched buffer (see supplementary movie S3).

Addition of exogenous PDE supplements the degradation of cAMP. In our experiments 


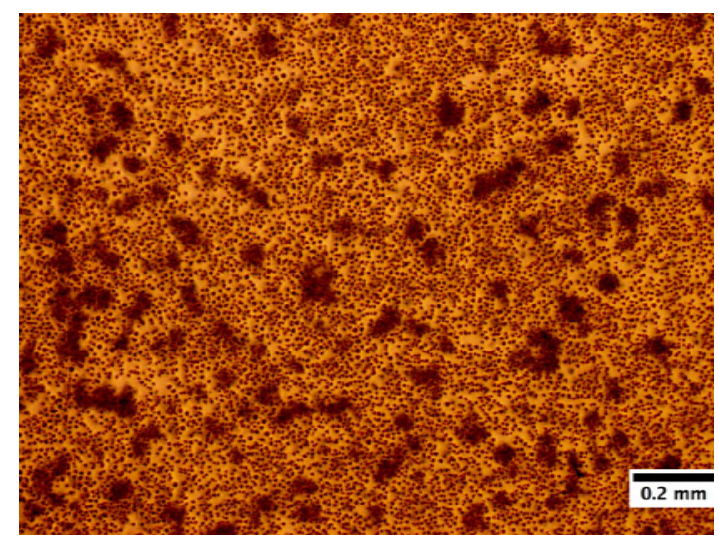

FIG. 7. The formation of aggregates in a mixture of a low-density $\left(200\right.$ cells $\left./ \mathrm{cm}^{2}\right)$ of haves and a very high density $\left(1 \times 10^{6}\right.$ cells $\left./ \mathrm{cm}^{2}\right)$ of have-nots. The haves act as centers of activity and the havenots around them aggregated. Due to the high degradation of cAMP by the PDE produced by the have-nots, the cAMP waves did not reach all the cells, due to which many cells were not included in any aggregate. This experiment was performed twice. Experiments with similar extreme ratios were performed about 12 times with similar results.

with PDE4A, we estimate the degradation time due to the exogenous PDE to range from 0.2 min to 130 min. (See Appendix A2 for details.) In accordance with our requirements, we observe tight aggregates only in cases where the degradation rate is smaller than or comparable to the wave period, as seen in figure 6 .

Having gained these insights, we return to the cooperation between the haves and the have-nots. The have-nots supply divalent ions and PDE (see supplementary note S1), both of which are vital for aggregation. In fact, in a mixture of a 200 cells $/ \mathrm{cm}^{2}$ of haves and 1 $\mathrm{x} 10^{6}$ cells $/ \mathrm{cm}^{2}$ of have-nots, so much PDE is produced that waves cannot propagate for long distances - cAMP is completely degraded at short distances (see supplementary movie S4 and figure 7), resulting in small aggregates, where, due to the absence of large scale waves, many cells were excluded. Thus, we see that the degradation rate is an important parameter for aggregation - both at very low and very high degradation rates, aggregation is not efficient.

We started this paper by naïvely called the wild-type cells the haves and the acaA- cells the have-nots, simply because the latter group is incapable of producing cAMP. But we found that the mutants provide other components that are vital for survival. While investigating their cooperation, we found that (1) a critical amount of divalent ions is necessary to 
keep the cells viable, and (2) PDE activity within a certain range is necessary for optimal aggregation. When these two criteria are satisfied, even a small fraction of cAMP producing cells can synchronize the population and cause aggregation, explaining the robustness of the mechanism. Cooperation between the cells occurs by the sharing of complementary resources. It would be interesting to see whether other species display such cooperation.

\section{METHODS}

\section{Preparation of the cells}

AX2 -214 cells and acaA ${ }^{-}$mutants (in AX3 background) were grown as Petri dish cultures in HL5 medium ( Formedium) at $22^{\circ} \mathrm{C}$. These cell lines were kind gifts from G. Gerisch and A. Kortholt respectivelt. The cells were harvested when they became confluent and were washed three times in phosphate buffer $\left(2 \mathrm{~g}\right.$ of $\mathrm{KH}_{2} \mathrm{PO}_{4}, 0.36 \mathrm{~g}$ of $\mathrm{Na}_{2} \mathrm{HPO}_{4} \cdot 2 \mathrm{H}_{2} \mathrm{O}$ per liter at $\mathrm{pH} 6$, autoclaved). The cells were then counted using a hemocytometer and diluted to the appropriate density with fresh phosphate buffer. They were then plated on a 8.6 $\mathrm{cm}$ diameter plastic Petri dish with $10 \mathrm{ml}$ phosphate buffer - this corresponds to a buffer thickness of $2 \mathrm{~mm}$. To conserve reagents, we sometimes used plastic Petri dishes divided into quadrants, or smaller diameter Petri dishes. In all these cases, we maintained a constant areal and volume density of the cells. Parafilm was wrapped around the dish lids to prevent evaporation of the buffer. To prevent condensation, we blew $24^{\circ} \mathrm{C}$ air above the dish lid. Development occurred in a dark room at $22^{\circ} \mathrm{C}$.

\section{Supernatants}

The supernatant was aspirated from the Petri dishes, and then centrifuged to pellet out any cells accidentally. The supernatant was then cleared using $0.2 \mu \mathrm{m}$ filters.

To fractionate the supernatant into the high and low molecular weight fractions, we used a $30 \mathrm{kDa}$ Amicon filter (EMD Millipore). The supernatant was added to the filter and spun down at it $3500 \mathrm{~g}$ for about $15 \mathrm{~min}$. This left about $200 \mu \mathrm{L}$ of high molecular weight retentate. The efflux contains the rest of the supernatant which has substances smaller than $30 \mathrm{kDa}$. To use the retentate as conditioning medium at the same concentration as in the supernatant, 
we reconstituted it in phosphate buffer of the same volume as the starting volume of the supernatant used.

The heat treatment consists of heating the supernatant uniformly at $80^{\circ} \mathrm{C}$ for about 20 min in a thermostat. The actual temperature was monitored using a thermometer. After $20 \mathrm{~min}$, the supernatant was allowed to cool to room temperature before being used for experiments.

\section{Addition of external PDE}

PDE4A were purchased from Enzo and Signal Chem. For each experiment, the enzyme was diluted based on the activity provided on sample and from the experimental value of the Michaelis-Menten constant for these enzymes. 1 unit $=1 \mathrm{nmol} / \mathrm{min}$ at $37^{\circ} \mathrm{C}$. For details of the calculations, see Appendix A2.

\section{ACKNOWLEDGEMENTS}

We are grateful to Maren S Müller and Katharina Gunkel for their invaluable support in the preparation of the cells and in procuring the chemicals and enzymes used in this study. We thank Bastian Hüllsmann and the Gorlich lab at the Max-Planck Institute for Biophysical Chemistry for their help and support in the use of their luminometer. We thank J. P. Sethna, V. Vogt, I. Guido and G. Gerisch for useful discussions. We thank C. Westendorf for help with the microscopes and discussions. This work has been supported by the Max Planck Society.

\section{COMPETING INTERESTS}

The authors declare no competing interests.

\section{SUPPLEMENTARY NOTE S1: MEASURING THE EXTRA-CELLULAR PDE AC- TIVITY}

To measure PDE activity, we used the PDELight kit from Lonza. The measurement works on the principle that PDE degrades cAMP to create AMP. The detection reagent provided 
in the kit converts AMP to ATP, Luciferin and oxygen. In the presence of luciferase, these combine to produce AMP and photons (among other products). The number of photons measured is thus an indicator of the amount of AMP present. We allowed the PDE from our cell populations to react with cAMP for $20 \mathrm{~min}$ and measured the resulting amount of AMP by counting photons with a luminometer. From the amount of AMP remaining, we estimated the degradation of the PDE in our sample.

We first collected the supernatant from the populations as described in Methods. We diluted the retentate of the filtration appropriately. For each sample, we created multiple dilutions of the retentate and added $30 \mu \mathrm{L}$ of each in a black 96 well plate. To each dilution, we added $10 \mu \mathrm{L}$ of $100 \mu \mathrm{M}$ cAMP. After 20 min of reaction time, we added the stop solution - a 1:1 mixture of the Lonza stop solution and $100 \mathrm{mM}$ DTT. Then, we added the detection reagent, and allowed $10 \mathrm{~min}$ for the reaction to stabilize. For each dilution, we created a background sample by adding the just diluted retentates without cAMP to the wells in the 96 well plate. During each experiment, for calibration, we added $30 \mu L$ of different concentrations of AMP to the same 96 well plate. To make the same volume, instead of cAMP, we added $10 \mu \mathrm{L}$ of phosphate buffer to these standards. We added the same amounts of stop solution and detection reagent. An integration time of $0.1 \mathrm{~s}$ was used. We recorded the luminosity every $10 \mathrm{~min}$ to account for the decay of AMP. We fit the time series of the intensities with a model (Appendix A3) to obtain the degradation rates.

We performed this assay to first find the degradation rate of cAMP in different cell populations. At high wild type cell densities, the degradation rate is about $0.007 / \mathrm{min}$ (figure 8 column a). At half this density, the degradation rate decreases to $0.0018 / \mathrm{min}$ (figure 8 column b) and at the low density, it is $0.0002 / \mathrm{min}$ (figure 8 column c). The mutants also produce a significant amount of PDE, although it is not as much as that produced by the same number of wild type cells (compare figure 8 columns a and d). Further, keeping the total cell density fixed, as the ratio of mutants to wild type cells is decreased, the population mixture produces more PDE (figure 8 columns d -f). From these measurements it was clear that the mutants contribute PDE in addition to the divalent ions in the mixture of low density wild types and high density mutants.

Next, to observe the effect of the heat-treated supernatant, we conditioned the low density WT cells with the heat treated supernatant, starved them for about six hours and then measured the PDE activity in the supernatant. We found that this activity (figure 9 column 


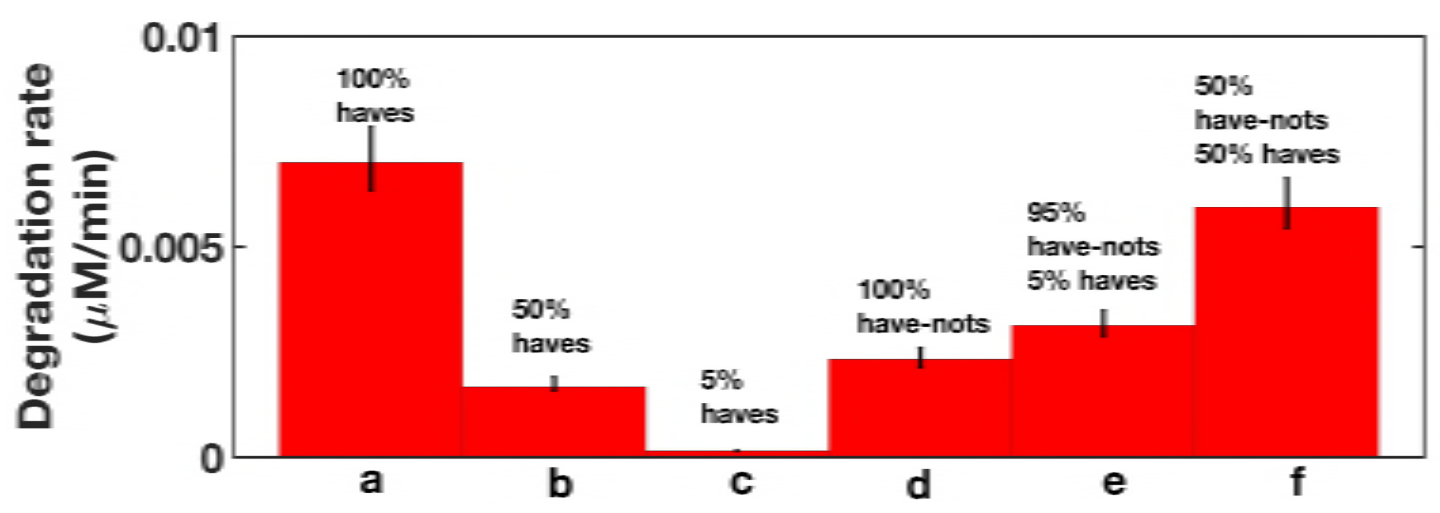

FIG. 8. Comparison of the degradation rate of the extra cellular PDE produce by different cell populations. $100 \%$ corresponds to a cell density of about $2 \times 10^{5}$ cells $/ \mathrm{cm}^{2}$. Experiments performed once.

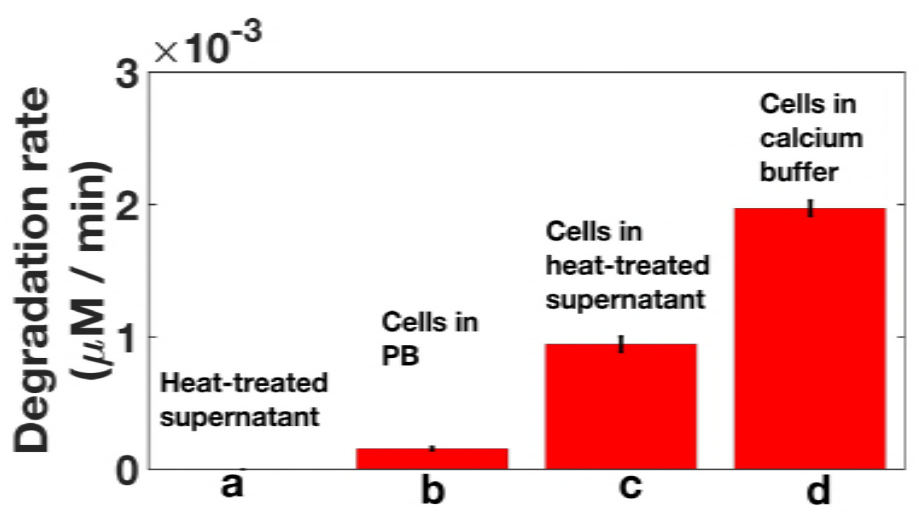

FIG. 9. Comparison of the degradation rate of the extra cellular PDE produced by the low density populations in different conditions. PB is phosphate buffer. Low density haves are used in columns b, c, and d. Experiments performed once.

c) was much higher than the activity of the low density WT cells in phosphate buffer (figure 9 column b). As a check, we verified that our heat treatment denatures PDE, column a in figure 9. Finally, the degradation rate was even higher in low density populations conditioned by the calcium enriched buffer (figure 9 column d) than in just phosphate buffer. This suggested that the addition of calcium, which keeps the cells viable, induces them to produce more PDE.

Our measurement of the reaction rates of the high density of haves is consistent with measurements by other groups [29]. However, both these rates correspond to a degradation time of about $100 \mathrm{~min}$. Theoretically, wave propagation is not possible at these rates. But 
we do observe waves. So there must be additional sources of degradation of cAMP that these measurements missed. What these could be, is an open question.

\section{APPENDIX}

\section{A1: The importance of PDE in long range signaling}

Consider the typical wild type cell density used in experiments i.e. cell density $\rho$ is $6 \mathrm{x}$ $10^{5}$ cells $/ \mathrm{ml}^{2}$. The typical cell separation $d_{\text {sep }}$ is given by

$$
d_{s e p}=\frac{1}{\sqrt{\rho}} .
$$

For the typical cell density mentioned above, this gives a $d_{\text {sep }}$ of about $13 \mu \mathrm{m}$. The typical wavelength of the cAMP waves is about $1-2 \mathrm{~mm}$. The dynamics of $D . d$. forms a reaction - diffusion system. If we assume that the degradation follows first order kinetics, the length scale $L$ for diffusion is set by the diffusion constant $D$ and the degradation rate of cAMP $\gamma$.

$$
L=\sqrt{\frac{D}{\gamma}}
$$

This gives,

$$
\gamma=\frac{D}{L^{2}}
$$

If we assume a diffusive length of about $130 \mu \mathrm{m}$, which corresponds to the distance between 10 cells $\left(10 \times d_{\text {sep }}\right)$, and use $D=0.024 \mathrm{~mm}^{2} / \mathrm{min}$, we get $\gamma=1.4 \mathrm{~min}^{-1}$, which corresponds to a degradation time of $0.7 \mathrm{~min}$. The diffusion length scale $L$ cannot be shorter than the typical separation between two cell, $d_{\text {sep }}$, because if it were, the signal would diffuse before it reached the neighboring cell, and there would be no excitation waves. Similarly, the characteristic decay time given by $1 / \gamma$ has to be shorter than the period of the waves ( 6 - $8 \mathrm{~min}$ ), so that cAMP can be degraded before the next wave begins. Therefore, the value of the degradation rate at high cell densities allows wave propagation because it is shorter than the period of the wave.

Now, the density of our low density population is about 70 times smaller than the value considered above. If we assume that the degradation scales linearly with density, the degradation rate of the low density population will be 70 times smaller, and the degradation time will be 70 times larger, or about $49 \mathrm{~min}$. This number is much higher than the period of the 


\section{A3: Obtaining the degradation rate}

We modeled the system in the following way. AMP is produced through the action of PDE, and there is a decay rate. For PDE produced by D.d.., it is known that the value of the Michaelis-Menten constant is $0.75 \mu \mathrm{M}$ [29]. In our experiments, we add a much higher concentration of cAMP. So the rate of the reaction can be assumed to be independent of the substrate concentration. This rate is also $V_{\max }$, the maximum rate of the reaction. We observe that AMP concentration decays after the reaction is stopped by adding the stop solution. Accounting for the production and decay of AMP, we can write

$$
\frac{d C}{d t}=k_{1}-k_{2} C
$$




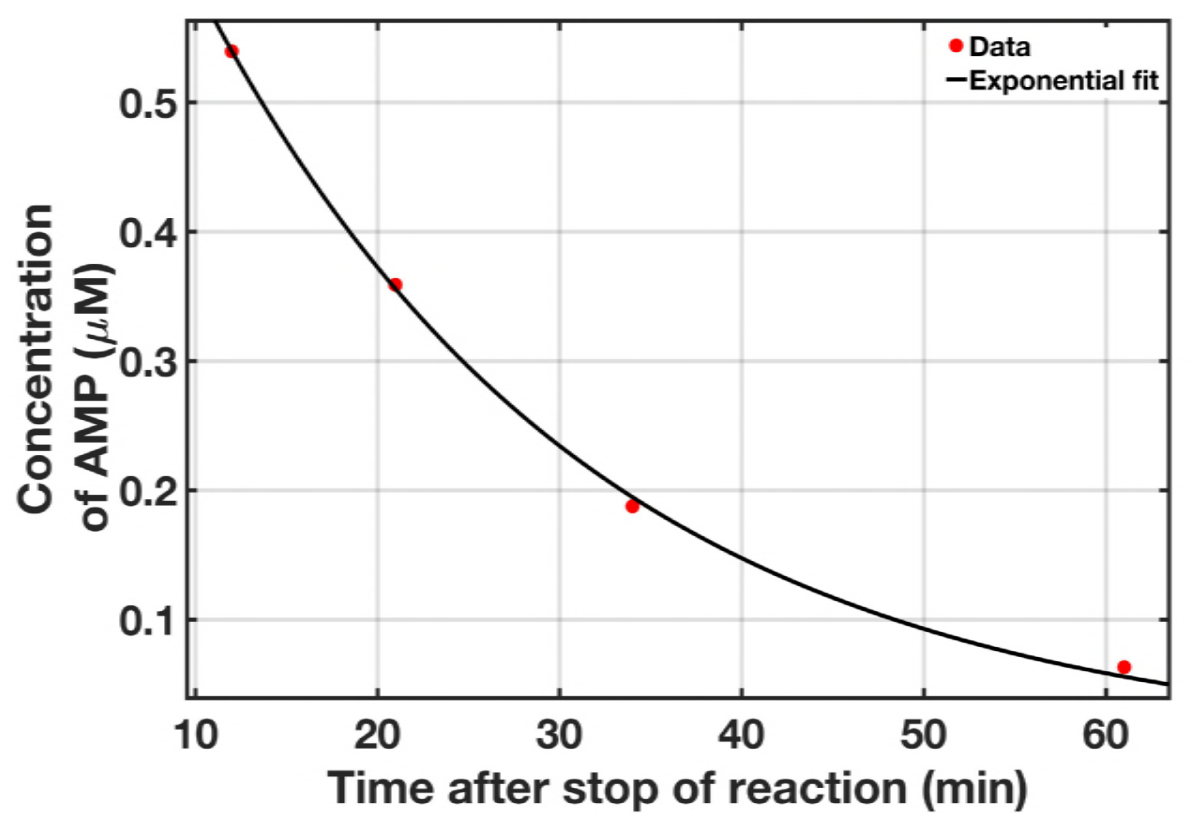

FIG. 10. Sample fit of the decay of the concentration of AMP as a function of time.

Here $\mathrm{C}$ is the concentration of AMP, $k_{1}$ is the degradation rate of PDE which creates AMP (this is the rate we are interested in finding) and $k_{2}$ is the decay rate of AMP. The solution to the equation is

$$
C(t)=\frac{k_{1}}{k_{2}}\left(1-\exp \left(-k_{2} t\right)\right)
$$

We stop the reaction after 20 min, the concentration of AMP after 20 min is $\mathrm{C}(20)$.

$$
C(20)=\frac{k_{1}}{k_{2}}\left(1-\exp \left(-20 k_{2}\right)\right)
$$

After these $20 \mathrm{~min}$, there is only decay of AMP because PDE doesn't act anymore. The equation for the concentration of AMP after these 20 min is therefore the decaying exponential

$$
C(t)=C(20) \exp \left(-k_{2} t\right)
$$

Here $\mathrm{t}$ is time after stopping the PDE action. We measure $\mathrm{C}(\mathrm{t})$ at different times after stopping. We first subtracted the background AMP and then converted the luminosities to concentrations using the AMP standards. Then we fit an exponential to the decaying concentration of AMP to get $\mathrm{C}(20)$ and $k_{2}$. We plug these values back into equation 6 to get the decay rate $k_{1}$. The error in $k_{1}$ is calculated by propagating the errors in $C(20)$ and $k_{2}$ from the confidence intervals provided by the fit. A sample fit is shown in the figure 10 . 
[1] K. B. Raper, Journal of Agricultural Research 50, 135 (1935).

[2] J. T. Bonner, American Journal of Botany 31, 175 (1944).

[3] G. Gerisch, Current Topics in Developmental Biology 3, 157 (1968).

[4] F. Alcantara and M. Monk, Microbiology 85, 321 (1974).

[5] P. N. Devreotes, M. J. Potel, and S. A. MacKay, Developmental Biology 96, 405 (1983).

[6] K. Tomchik and P. Devreotes, Science 212, 443 (1981).

[7] P. C. Newell, "Aggregation and cell surface receptors in cellular slime molds," in Microbial Interactions, edited by J. L. Reissig (Springer US, Boston, MA, 1977) pp. 1-57.

[8] D. Dormann and C. J. Weijer, Development 128, 4535 (2001).

[9] D. M. Veltman, I. Keizer-Gunnik, and P. J. Van Haastert, The Journal of Cell Biology 180, $747(2008)$.

[10] B. Wang and A. Kuspa, Science 277, 251 (1997).

[11] S. Dolatabadi, J. Candia, N. Akrap, C. Vannas, T. Tesan Tomic, W. Losert, G. Landberg, P. Åman, and A. Ståhlberg, Frontiers in Genetics 8, 1 (2017).

[12] F. Siegert and C. Weijer, Journal of Cell Science 93, 325 (1989).

[13] C. Wang, S. Chowdhury, M. Driscoll, C. A. Parent, S. K. Gupta, and W. Losert, Journal of The Royal Society Interface 11 (2014), 10.1098/rsif.2014.0684.

[14] E. Décavé, D. Rieu, J. Dalous, S. Fache, Y. Bréchet, B. Fourcade, M. Satre, and F. Bruckert, Journal of Cell Science 116, 4331 (2003).

[15] P. R. Fisher and Z. Wilczynska, FEMS Microbiology Letters 257, 268 (2006).

[16] D. F. Lusche, D. Wessels, and D. R. Soll, Cell Motility and the Cytoskeleton 66, 567 (2009).

[17] F. T. Marin and F. G. Rothman, The Journal of Cell Biology 87, 823 (1980).

[18] R. Gomer, I. Yuen, and R. Firtel, Development 112, 269 (1991).

[19] I. S. Yuen, R. Jain, J. D. Bishop, D. F. Lindsey, W. J. Deery, P. J. Van Haastert, and R. H. Gomer, The Journal of Cell Biology 129, 1251 (1995).

[20] R. Jain, I. S. Yuen, C. R. Taphouse, and R. H. Gomer, Genes \& Development 6, 390 (1992).

[21] F. Alcantara and G. W Brazill, Journal of general microbiology 92, 351 (1976).

[22] V. Riedel, D. Malchow, G. Gerisch, and B. Nägele, Biochemical and Biophysical Research Communications 46, 279 (1972). 
[23] G. Gerisch, D. Malchow, V. Riedel, E. Müller, and M. Every, Nature 235, 90 (1972).

[24] R. H. Kessin, S. J. Orlow, R. I. Shapiro, and J. Franke, Proceedings of the National Academy of Sciences of the United States of America 76, 5450 (1979).

[25] M. Coukell and A. Cameron, Journal of Cell Science 90, 691 (1988).

[26] M. B. Coukell and A. M. Cameron, Journal of Cell Science 97, 649 (1990).

[27] G. Europe-Finner, S. McClue, and P. Newell, FEMS Microbiology Letters 21, 21 (1984).

[28] F. Yamasaki and H. Hayashi, The Journal of Biochemistry 92, 1911 (1982).

[29] S. Bader, A. Kortholt, and P. J. M. Van Haastert, Biochemical Journal 402, 153 (2007).

[30] R. Sucgang, C. J. Weijer, F. Siegert, J. Franke, and R. H. Kessin, Developmental Biology 192, 181 (1997).

[31] G. L. Garcia, E. C. Rericha, C. D. Heger, P. K. Goldsmith, and C. A. Parent, Molecular Biology of the Cell 20, 3295 (2009).

[32] K. Omori and J. Kotera, Circulation Research 100, 309 (2007). 